\title{
La mediación social en la apropiación de una nueva propuesta para la alfabetización inicial
}

\author{
Epifanio Espinosa Tavera \\ Universidad Pedagógica Nacional \\ Ruth Mercado Maldonado \\ DIE-CINVESTAV
}

\section{Resumen}

Este artículo muestra la importancia de la mediación social en los procesos de apropiación, por parte de los docentes, de nuevas propuestas para la enseñanza derivadas de las reformas. Se muestra la apropiación como un proceso social activo en el que los maestros construyen y reelaboran sentidos y saberes a partir del uso de las nuevas herramientas que les son propuestas. Desde una perspectiva etnográfica se describen algunas de las formas y significados mediante los cuales las docentes del estudio se apropian de una nueva propuesta pedagógica introducida a nivel nacional para la alfabetización inicial. Se identifica cómo, al interactuar con la propuesta, las profesoras desarrollan interpretaciones y prácticas propias que no corresponden a la reproducción puntual de la misma, sino a un proceso de apropiación. Ésta también es producto de una construcción colectiva entre las docentes, pues quienes intentan poner en práctica la propuesta comparten sus reflexiones, dudas y decisiones con otras profesoras que ya la han experimentado, como la maestra "asesora" y otras colegas de la misma escuela. El análisis tiene como referencia las experiencias descritas por maestras de primer grado en escuelas mexicanas al participar en un programa de asesoría derivado de la reforma que dio lugar al Programa Nacional para el Fortalecimiento de la Lectura y la Escritura en la Educación Básica (PRONALEES).

\section{Palabras clave}

Reforma educativa - Apropiación - Mediación social - Saberes docentes.

Correspondência: 


\title{
Social mediation in the appropriation of a new proposal for initial literacy
}

\author{
Epifanio Espinosa Tavera \\ Universidad Pedagógica Nacional \\ Ruth Mercado Maldonado \\ DIE-CINVESTAV
}

\begin{abstract}
This article aims at showing the importance of social mediation in the processes of appropriation by teachers of new teaching proposals derived from the reforms. Appropriation is depicted as an active social process in which teachers construct and redefine senses and knowledges from the use of the new tools placed before them. An ethnography-based description is made of some of the forms and meanings through which the teachers in this investigation appropriate a new pedagogical proposal for initial literacy introduced at the national level. It was possible to identify how in their interaction with the proposal, the teachers develop their own interpretations and practices which, rather than corresponding to a point-by-point reproduction of the proposal, constitute a process of appropriation. The latter also results from a collective construction by the teachers, since those who try to put the proposal in practice share their reflections, doubts, and decisions with other teachers that have already done it before, such as the "consulting" teacher and other colleagues from the same school. The analysis refers to the experiences described by teachers working in first grade classes of Mexican schools who were taking part in an advisory program derived from the reform that gave rise to the National Program for the Improvement of Reading and Writing in Basic Education (PRONALEES).
\end{abstract}

\section{Keywords}

Education reform - Appropriation - Social mediation - Teacher knowledges.

Contact:

Epifanio Espinosa Tavera

Clemente Mejía, 35

Col. Estado de Guerrero

C.P. 39480 - Acapulco, Gro. - MX

E-mail: espitave@hotmail.com 
En 1993 en México se impulsó una reforma de la educación básica. Se produjeron transformaciones curriculares con el propósito de propiciar en los alumnos el desarrollo del pensamiento reflexivo y de competencias para el uso efectivo en la vida cotidiana de conocimientos y habilidades para la resolución de problemas y la comunicación oral y escrita, entre otros.

No obstante el consenso sobre la pertinencia de estos cambios, su incorporación a la trama cultural y las prácticas escolares continúa siendo un campo abierto al debate y la acción. Estudios del cambio educativo muestran la enorme complejidad que media entre las propuestas de las reformas y su funcionamiento concreto en las escuelas. Por un lado, los procesos seguidos tanto en la formulación como en la implementación misma de las reformas, definen en buena medida las posibilidades de su incorporación a las prácticas (Fullan; Stiegelbahuer, 1997). Por otro lado, se acepta cada vez más el peso histórico y cultural de las formas de organización y funcionamiento de las escuelas en las posibilidades y límites transformadores de las reformas (Ezpeleta, 2004; Hargreaves, 1996; Tyack; Cuban, 2000; Viñao, 2001).

Actualmente es lugar común señalar que los maestros son claves en la transformación de la enseñanza. Este reconocimiento ha propiciado la realización de diversos estudios relacionados con la puesta en práctica de las reformas por los maestros.

Algunos estudios han enfocado los usos que hacen los maestros de las nuevas propuestas y materiales para la enseñanza (p.e. Avila, 2001; Carvajal, 2001). Generalmente, estos estudios destacan el grado, o los aspectos de las nuevas propuestas que entran o no en las prácticas de enseñanza, así como las dificultades que los maestros enfrentan para su empleo.

En otra línea, la formación de docentes, se indagan los efectos de algunos programas de formación diseñados para que los maestros adquieran conocimientos y concepciones que se consideran fundamentales para la implementación de los cambios promovidos con las reformas (Avalos, 1996; Tatto, 1999). Las principales búsquedas y hallazgos aluden a la pertinencia de los materiales y su diseño, o a la implementación y su relación con los resultados - esperados o no - del proceso formativo.

Desde una mirada distinta, en este artículo buscamos aportar a la comprensión de las formas en que los maestros se apropian de nuevas propuestas de enseñanza. Presentamos resultados parciales de un estudio más amplio en el que indagamos sobre las condiciones y procesos que median en dicha apropiación.

Partimos del supuesto de que es en la enseñanza donde los maestros construyen saberes sobre su trabajo (Mercado, 2002; Rockwell; Mercado, 1986; Olson, 1992; Schön, 1992, Tardiff, 2004). Nuestra búsqueda enfoca las prácticas de enseñanza y las trayectorias seguidas por maestros en sus acercamientos a una de las propuestas de enseñanza promovidas por la actual reforma: la de alfabetización inicial instrumentada a través del Programa Nacional para el Fortalecimiento de la Lectura y la Escritura en Educación Básica (PRONALEES). Trabajando con maestras que valoran y emplean intensivamente tal programa, procuramos comprender, desde su propia perspectiva, los procesos por los que han transitado, así como sus razones para usar esos materiales tal como lo hacen.

En este artículo enfocamos la importancia de la mediación social en la apropiación de nuevas propuestas pedagógicas. Mostramos la apropiación como un proceso social activo en el que los maestros construyen y reelaboran sentidos y saberes sobre la enseñanza a partir del uso de las nuevas herramientas que se les proponen. La mediación social acontece de formas diversas y en múltiples niveles en los procesos de apropiación. En nuestro estudio identificamos, por ejemplo, que los maestros realizan en el aula usos plurales y móviles (Chartier, 1995) del PRONALEES que resultan de la dialogía con múltiples voces sociales (Bajtin', 1989) - los padres, los niños, el currículo escolar, las condiciones de trabajo, la propia institución

1. En las publicaciones en portugués y francés el nombre de este autor ruso se escribe Mikhail Bakhtin. En las obras escritas en español, en cambio, es más común encontrarlo como Mijail Bajtin. 
entre otras. En este artículo analizamos específicamente algunas de las formas y sentidos en que la interacción con personas más conocedoras de la propuesta, como la maestra asesora, y con otros profesores colegas que también experimentaban su uso, posibilitaron el acceso de las maestras del estudio a la propuesta del PRONALEES. El análisis tiene como referentes las experiencias descritas por las maestras acerca de su participación en un programa de asesoría y de sus interacciones con otros colegas.

\section{ei PRONALeEs}

El PRONALEES, vigente de 1995 a 2002, emprendió la reformulación curricular de programas y materiales para la enseñanza de español de la escuela primaria. Su propuesta de alfabetización, aún vigente, adoptó el enfoque comunicativo y funcional, planteado desde la reforma curricular de 1993. La alfabetización desde tal enfoque pretende desarrollar competencias para el uso efectivo del lenguaje escrito en diversas situaciones y propósitos comunicativos de la vida cotidiana.

En el PRONALEES la alfabetización inicial en los primeros grados se concibe articulada a la producción e interpretación de textos con diferentes propósitos. Así, en el programa de estudios para el primer grado la producción de textos por parte de los niños es una actividad frecuente. Desde las primeras lecciones se propone, por ejemplo, que los niños escriban palabras ya sea para completar algún texto, elaborar alguna lista, o etiquetar materiales. Los textos van aumentando en diversidad y complejidad a lo largo del curso. En las últimas lecciones se propone que los niños participen en la elaboración de cuentos, cartas, recados, anuncios, carteles o invitaciones. Asimismo, se enfatiza desde primer grado el desarrollo de la comprensión lectora. A los maestros se les recomienda implementar varias modalidades de lectura (lectura en voz alta, lectura compartida, lectura guiada, lectura por parejas y lectura independiente), así como actividades que pueden realizar antes, durante y después de la lectura con el objeto de favo- recer en los niños una relación de sentido y comprensión con lo que leen (SEP, 1998). Se considera que con estas experiencias y la reflexión sistemática sobre los escritos que se producen y se leen, los niños comprenderán tanto el funcionamiento alfabético convencional de la escritura como la diversidad de sus usos y funciones sociales.

La propuesta del PRONALEES no es totalmente novedosa en el medio educativo mexicano. Desde 1980, la Dirección General de Educación Especial emprendió diversas iniciativas para promover una propuesta similar con fundamentos constructivistas denominada Propuesta para el Aprendizaje de la Lengua Escrita (PALE). Inicialmente se impulsó como una medida remedial para alumnos de primero y segundo grados con antecedentes o en riesgo de repetición, posteriormente se buscó incorporarla a las prácticas de enseñanza de esos grados, para lo cual se conformaron equipos de profesores que asesoraban ${ }^{2}$ a los maestros en su implementación. La última de estas iniciativas fue la Propuesta para la Adquisición de la Lengua Escrita y las Matemáticas (PALEM), la cual se promovió en el estado donde se realizó el estudio desde 1990 hasta 1997, cuando surgió el PRONALEES.

Las diversas iniciativas fueron oficialmente impulsadas por la SEP, sin embargo, la del PALEM existió al margen de los programas y libros de texto de los alumnos y su implementación dependía de la aceptación voluntaria de los maestros. El enfoque de alfabetización promovido por esa propuesta se introdujo al currículo oficial con la reforma de 1993, y fue hasta 1997, con el PRONALEES, que se diseñaron programas y libros para los alumnos alineados a dicho enfoque.

2. En México, los asesores son profesores con diversos niveles de experiencia en la enseñanza que generalmente son capacitados para proporcionar apoyo técnico pedagógico a los demás maestros en la instrumentación de algún programa o proyecto educativo. Mientras se desempeñan como asesores los maestros son liberados de la docencia. Las asesorías del PRONALEES, en la práctica, se realizaron en distintas condiciones y asumieron sentidos diversos, propiciando distintas posibilidades de apoyo real para los maestros en su apropiación de la propuesta (Espinosa et al., 2003). En el estado donde se realizó el estudio, esta labor de asesoría se inició en 1987 con el PALEM y se mantuvo vigente con el PRONALEES hasta el año 2002. 
Los supuestos del PRONALEES acerca de la enseñanza y el aprendizaje del lenguaje escrito presentan diferencias significativas con las prácticas y creencias de los maestros del primer grado. Tradicionalmente, las prácticas dominantes de alfabetización inicial en la escuela primaria han enfatizado la adquisición del código alfabético como el aspecto fundamental del aprendizaje del lenguaje escrito. En esta perspectiva se enseñan una por una las letras del alfabeto, su sonido, las sílabas que con ella se forman, y su ejercitación en la escritura de palabras. En esta lógica de trabajo, la producción e interpretación de textos se abordan posteriormente a la adquisición del código alfabético, cuando los niños ya 'saben leer y escribir. En este sentido, la ausencia en los materiales del PRONALEES de una estrategia para la enseñanza explícita de las letras y la proposición de que los alumnos participen en la lectura y producción de textos desde el inicio, son situaciones que, como se verá más adelante, inicialmente resultan incomprensibles y carentes de sentido para los maestros.

\section{Características del estudio}

El estudio se realizó desde una perspectiva etnográfica; entendiendo la etnografía como una metodología que busca dar cuenta de los procesos locales y de los sentidos sociales que en ellos se expresan y movilizan (Geertz, 1987; Rockwell, 1987). En este sentido, conocer el trabajo que las maestras de nuestro estudio realizan con los materiales del PRONALEES, las razones por las cuales los emplean y la manera en que llegaron a valorarlos como herramienta útil para la alfabetización de sus alumnos, es fundamental para comprender, desde su propia perspectiva, cómo es que una propuesta inicialmente poco clara e incierta para ellas llegó a ser incorporada como herramienta valiosa para la enseñanza.

Trabajamos en 4 escuelas de colonias de la periferia de una ciudad de provincia en uno de los estados con mayor rezago educativo de México. Las condiciones de pobreza y marginación de las colonias es un común denominador de estas escuelas, aunque es más patente en dos de ellas. En el camino a estas escuelas se atraviesa por calles pavimentadas en algunos tramos, la mayor parte es de terracería. Por la mayoría de las calles escurren aguas negras, cuya pestilencia se mezcla con el mal olor del excremento de diferentes animales. Sólo una escuela, la de Laura, se ubica en una colonia que cuenta con mayores servicios. Es una gigantesca unidad habitacional de interés social para trabajadores, todas sus calles están pavimentadas, cuenta con servicio de drenaje, áreas verdes, canchas deportivas y varias escuelas de preescolar, primaria y secundaria.

La asistencia previa de los niños al preescolar es variable en estas colonias. En ninguno de los grupos de primer grado del estudio los niños contaban en su totalidad con experiencia preescolar, y en dos casos - los grupos de Macrina y Perla - la proporción de estos niños era menos de la mitad.

Ante la distancia muy marcada entre la propuesta de alfabetización del PRONALEES y las prácticas más comunes de los maestros de los primeros grados, así como la persistencia de estas prácticas ante reformas anteriores, optamos por trabajar con maestros que emplearan y valoraran positivamente la propuesta del PRONALEES. Examinar las prácticas de estos maestros y las formas en que fueron incorporando el PRONALEES a la enseñanza, nos pareció una vía adecuada para comprender los procesos de apropiación.

Cinco maestras aceptaron colaborar en el estudio. Tres eran de primer grado y dos trabajaban con segundo. También participó una maestra (Nora), que fue asesora en la implementación de la PALEM y del PRONALEES.

Cuatro maestras trabajaron previamente con la PALEM apoyadas por Nora. Ellas remiten a este periodo sus principales descubrimientos y aprendizajes en el trabajo con PRONALEES. La otra maestra (Macrina) conoció el PRONALEES durante su implementación con apoyo de otro asesor.

Las maestras contaban con varios años en la docencia (de 11 a 30 años), y habían 
CUADRO 1. Características de las maestras del estudio.

\begin{tabular}{|c|c|c|c|c|c|c|c|}
\hline \multirow{2}{*}{ Maestra } & \multirow{2}{*}{ Estudios } & \multirow{2}{*}{$\begin{array}{c}\text { Grado } \\
\text { (alumnos) }\end{array}$} & & \multicolumn{3}{|c|}{ Años de } & \multicolumn{2}{|c|}{ Inició de trabajo con } \\
\cline { 4 - 8 } & & & & $\begin{array}{c}\text { trabajo con } \\
\text { ergrado }\end{array}$ & asesoría & PALEM & PRONALEES \\
\hline Paola* & $\begin{array}{l}\text { Normal Básica } \\
\text { UPN*** }\end{array}$ & $1^{\circ}(25)$ & 20 & 3 & 1 & $1993-94$ & $2000-01$ \\
\hline Perla* & $\begin{array}{l}\text { Normal Básica } \\
\text { UPN }\end{array}$ & $1^{\circ}(23)$ & 17 & 5 & 3 & $1990-91$ & $1997-98$ \\
\hline Laura* $^{*}$ & $\begin{array}{l}\text { Normal Básica } \\
\text { UPN }\end{array}$ & $2^{\circ}(26)$ & 11 & 3 & 1 & $1990-91$ & $2001-02$ \\
\hline Sofia* & Normal Básica & $2^{\circ}(18)$ & 30 & 5 & 5 & $1990-91$ & $1997-98$ \\
\hline Macrina & Normal Básica & $2^{\circ}(16)$ & 18 & 2 & 3 & - & $1999-00$ \\
\hline
\end{tabular}

* Maestras asesoradas por Nora.

** Estudios de licenciatura en la Universidad Pedagógica Nacional.

trabajado en al menos tres ocasiones con el primer grado utilizando materiales de la PALEM o del PRONALEES. Al momento del estudio tres de ellas habían cursado la licenciatura en educación y dos sólo tenían la normal básica.

Nora, la asesora del PRONALEES, tenía 29 años en la docencia al realizarse el estudio y tenía una amplia formación profesional: normal básica, dos especializaciones en la normal superior geografía e historia -, licenciatura en educación, una maestría en ciencias de la educación y era candidata a doctora. Ella fue asesora de 1990 a 2001 aproximadamente, primero de la PALEM y después del PRONALEES. Además de docente y directora en el nivel primario, Nora ha trabajado en el nivel medio superior, en varias instituciones de formación de maestros, y trabaja, desde inicios de los noventa, en la Universidad Pedagógica Nacional.

Nora quiso ser asesora del PALEM por el conocimiento que tenía de la concepción psicogenética de adquisición de la escritura y por el uso ocasional que hizo de la propuesta en su propia escuela. Le entusiasmó la respuesta de los niños y consideró que podía aprender más sobre la propuesta al mismo tiempo que apoyaba a otros maestros a implementarla. Su conocimiento de la propuesta y sobre todo de su instrumentación práctica era poco, fue aprendiendo junto con los maestros. Las maestras del estudio, por su parte, le atribuyen un amplio conocimiento de la propuesta y valoran de manera sobresaliente su disposición para apoyarlas y la confianza que les inspiraba para comentarle sus dudas y problemas. Aunque
Nora logró la adscripción de todo su tiempo laboral en la UPN, por lo que abandonó el PRONALEES, varias maestras continuaron frecuentándola ya sea para consultarle alguna situación particular o para exponerle las novedades que emprendían.

Durante los más de 10 años, Nora trabajó en una misma zona escolar ${ }^{3}$ y asesoró fundamentalmente a los docentes de primer grado - de dieciocho a veintidós profesores; aunque también ayudaba a los de segundo cuando se lo solicitaban. Su modalidad de asesoramiento contemplaba tres tipos de acciones. Al inicio del año escolar realizaba un curso para que los maestros conocieran la propuesta, los materiales, y sus supuestos sobre el aprendizaje, además de realizar una planeación inicial. En segundo lugar, se reunía mensualmente con todos los maestros de la zona para hacer la planeación y cada dos meses para presentar la evaluación de resultados. Nora comenta que descubrió que al inicio del año se presentaban las mayores dudas y temores de los maestros sobre la propuesta, por lo que, con el respaldo del supervisor, decidió que las reuniones fueran quincenales durante los dos primeros meses. En tercer lugar, visitaba a los maestros a lo largo de todo el año, conversaba con ellos sobre su trabajo y sus dudas y, en ocasiones, trabajaba directamente con los niños, principalmente en la realización de evaluaciones.

3. En México las zonas escolares son demarcaciones administrativas de un conjunto de escuelas, aproximadamente diez. Cada zona escolar está a cargo de un supervisor, un profesor con experiencia en la docencia y la dirección de escuelas primarias. 
La dinámica de esta asesoría suscitó la actuación conjunta de Nora y los maestros en la resolución compartida de tareas como la planeación, la evaluación y la búsqueda de soluciones a los problemas enfrentados durante la implementación de la propuesta - primero de la PALEM y luego del PRONALEES. Este proceso, como se verá más adelante, posibilitó el acceso de las maestras del estudio a la propuesta y a los saberes necesarios para su empleo como herramienta útil en la alfabetización de los niños.

El trabajo de campo se realizó a lo largo del ciclo escolar 2001-2002. Se visitó a cada maestra en al menos 6 ocasiones. En las visitas observamos el trabajo que hacían con los niños y el uso que daban a los materiales del PRONALEES. Asimismo, conversamos ampliamente con ellas a propósito de situaciones y experiencias que comentaban, así como de sus experiencias previas de trabajo con el primer grado y de su participación anterior en la PALEM y el PRONALEES. Generalmente, durante las clases, las maestras comentaban espontáneamente sobre aspectos que consideraban importantes: por qué estaban haciendo así el trabajo, hacían comparaciones con lo hecho en otras ocasiones, señalaban dificultades que apreciaban en los niños y las iniciativas que emprendían. Asimismo, se referian constantemente a las oportunidades de aprendizaje que les brindó la asesoría durante la implementación de la PALEM. Adicionalmente, se realizaron varias conversaciones breves y tres entrevistas amplias con Nora, la asesora del PRONALEES. Las clases y las conversaciones fueron audio y videograbadas, y posteriormente fueron transcritas en versiones ampliadas. El archivo contiene 59 registros ampliados transcritos (47 contienen observaciones más entrevistas y 12 son sólo entrevistas).

\section{Apropiación, acceso y mediación social desde la perspectiva sociocultural}

Una de las empresas de la perspectiva sociocultural es comprender las formas en que las personas se apropian de las prácticas sociales, cómo llegan a constituirse en participantes plenos de las mismas. Mientras las teorías clásicas conciben el aprendizaje como proceso individual que acontece en la mente de las personas, para la perspectiva sociocultural la apropiación es un proceso social que se despliega y transcurre mediante la participación misma de las personas en las prácticas sociales, por lo que apropiación de y participación en las prácticas sociales no son momentos diferenciados, sino aspectos de un mismo proceso (Lave; Wenger, 2003; Pea, 2001; Wenger, 2001).

La participación como constructo analítico para dar cuenta de la apropiación de las prácticas sociales, alude simultáneamente al involucramiento activo de las personas, pero también a la interacción con las demás personas implicadas en su realización (Lave; Wenger, 2003; Wenger; 2001). Las diversas oportunidades de participar, en este doble sentido, ofrecen distintas posibilidades de acceso a las prácticas sociales, de forma que sus propósitos y los procesos para lograrlos, inicialmente "ocultos" a los ojos de los principiantes, van cobrando mayor "transparencia".

Las herramientas que median en el desarrollo de las prácticas para la consecución de los objetivos que persiguen las personas intervienen en este proceso "aportando" la inteligencia (Pea, 2001), los escondrijos de información (Lave, 2001), o los "residuos cognitivos" (Cole; Engestrom, 2001), que en aquéllas se "han cristalizado", mediante su intervención y empleo en acciones anteriores. La contribución de tales aportes al logro de los propósitos y la manera de incorporarlos a la acción - las maneras de emplear en la acción las herramientas -, sin embargo, no son evidentes a partir de la herramienta misma. Desde la perspectiva sociocultural (Hutchins, 2001; Pea, 2001), las herramientas adquieren una significación en su empleo en actividades concretas y específicas orientadas a propósitos particulares. Es decir, los "aportes" de las herramientas, o más bien lo que ellas pueden aportar a la realización de una tarea y al logro 
de sus metas, se construye en la actuación misma; en este sentido, la implicación en las actividades de una práctica, posibilita a las personas el acceso a los sentidos y los usos posibles de sus herramientas.

Al participar en el desarrollo de la práctica las personas entran en contacto con las tecnologías empleadas en su realización, aprecian cómo éstas contribuyen a la consecución de los propósitos, las posibilidades de sus usos y las maneras de emplearlos en circunstancias específicas y cambiantes, de forma que contribuyan a los objetivos de la empresa (Pea, 2001; Hutchins, 2001; Cole, 1999). Estos planteamientos permiten concebir la apropiación de nuevas propuestas pedagógicas como un proceso que tiene lugar en el contexto de su uso para la realización cotidiana de la enseñanza. Concebimos la utilización de los recursos que sugieren las nuevas propuestas, en las condiciones particulares en que realizan su trabajo, como el contexto donde los maestros construyen sentidos sobre su utilidad y pertinencia para conducir la enseñanza. En este proceso, ellos elaboran comprensiones acerca de los aportes de dichos recursos al aprendizaje de sus alumnos y producen saberes prácticos relativos a las maneras en que pueden emplearlos de forma que contribuyan al logro de los objetivos que se proponen.

Según proponen estos autores, las demás personas con las que se comparte una misma empresa actúan como mediadores en el acceso a los saberes específicos y necesarios para intervenir de manera efectiva en las prácticas. Este proceso transcurre mediante la interacción social suscitada por la participación conjunta en la realización de las prácticas.

Por una parte, la observación o la actuación conjunta con los prácticos expertos en diversas tareas, provee a los principiantes de una visión de conjunto del funcionamiento de las prácticas, de los medios que pueden ser empleados y los modos específicos de emplearlos ante situaciones particulares. En un sentido vygotskiano, las personas más informadas actúan como mediadores sociales en la apropiación de las prácticas y sus tecnologías actuando como guías, como andaderas sociales para los principiantes (Vygotski, 1988). La participación con los más experimentados propicia el acceso de los "iniciados" a los sentidos y saberes que se expresan y movilizan en el desarrollo de la práctica. De acuerdo con Pea (2001):

Los aportes de muchos sistemas de registros son en gran medida culturales en el siguiente sentido: es indispensable que una persona haya sido introducida en las actividades que dan significado a esas inscripciones [...] antes de esa iniciación, las convenciones y el empleo de las inscripciones son por lo común oscuros. Los usuarios maduros de un sistema de registro conocen los tipos de tareas para las cuales el sistema es útil [...]. Gran parte de eso es imperceptible para el iniciado, puesto que tal práctica social no está $<<$ en $>>$ la representación misma, sino en sus funciones relacionadas con las actividades que las personas desarrollan en el mundo. (p. 94)

La interacción entre los aprendices, por otra parte, constituye también una mediación social importante en la apropiación de las prácticas sociales y de nuevas herramientas (Wenger, 2001). Aunque persigan una misma empresa, las personas enfrentan heterogéneas situaciones ante las cuales construyen y reelaboran significados y saberes sobre la práctica. Las personas, en este sentido, son portadoras de un saber específico, de fondos sociales de conocimiento que circulan entre ellas al interactuar sobre la base de tareas y problemas comunes. Consideramos, en este sentido, la apropiación de nuevas propuestas pedagógicas como un proceso social situado en la práctica de la enseñanza. En este marco, las personas más informadas sobre aquéllas, como en el caso de este estudio lo fue la asesora de PRONALEES, y los maestros involucrados en su implementación median socialmente unos con otros en su apropiación. La interacción entre los maestros, a propósito de 
las tareas que enfrentan en la enseñanza, propicia la circulación y construcción compartida de sentidos y saberes sobre los nuevos recursos que se les proponen. En las siguientes dos secciones mostramos parte de este proceso promovido y alentado en el contexto de una de las asesorías promovidas por el PRONALEES.

\section{Mediación social y construcción de sentidos sobre la contribución al aprendizaje de nuevos recursos}

Las maestras del estudio decidieron incorporar en sus clases recursos de la PALEM o del PRONALEES por diversas razones: por el trabajo que observaban de algunos colegas, por los contenidos revisados en programas de desarrollo profesional, o porque contarían con asesoría o el apoyo de una o de algún colega al que le reconocian experiencia y conocimiento. Que tomaran esta decisión no implicó necesariamente que tuvieran un pleno conocimiento de las propuestas y de sus supuestos, de los propósitos que animan las estrategias didácticas ahi sugeridas. Más bien, según manifestaron, tenían dudas sobre su efectividad y temían que no lograran alfabetizar a los niños; particularmente se sentían contrariadas por algunas de las actividades sugeridas en las propuestas y se cuestionaban si estaban utilizando de manera adecuada los recursos.

Inicialmente, las maestras compartían algunas de las ideas sostenidas en esas propuestas - cuestionar a los niños, hacerlos reflexionar, que comprendan lo que leen. No obstante, distantes de sus creencias y prácticas de enseñanza, los nuevos recursos les resultaban confusos y poco comprensibles en cuanto a su utilidad para la enseñanza y el aprendizaje inicial de la escritura.

En PRONALEES, por ejemplo, se sugiere involucrar a los niños en la producción e interpretación de textos desde el inicio de la escolarización. Para muchos maestros esta es una proposición inconcebible, pues entra en contradicción con la práctica más o menos generalizada en el primer grado de la escuela primaria de iniciar con la enseñanza de sílabas y palabras, según las letras que van trabajando en forma secuencial.

En la perspectiva de muchos maestros, la lectura y escritura se abordan después de la adquisición del código alfabético, por ello, el hecho de que los libros de PRONALEES propongan desde el inicio que los niños participen en la lectura de un texto y realicen actividades de escritura, suele conducir a algunos maestros a considerar que estos materiales son apropiados para niños que ya saben leer y escribir, pero no para quienes están iniciando en la escuela este aprendizaje, como sugería Macrina:

Le digo a la maestra Sonia: "estos libros de primer año están fabulosos pero para un niño que ya viene leyendo y escribiendo, para este tipo de niños de aquí, no". Porque Sonia me decía: "Macri mi hijo ya va al primer año y ya sabe leer y escribir, qué va hacer en primero". Yo le dije: "el libro de español lo va a completar tan perfectamente, que el maestro de primero ya no va a tener problemas, porque ahí (en el libro) le están manejando 'escribe tu nombre', 'escribe el nombre de tu mamá y de tu papá'. Entonces él va a escribir a la perfección y no va haber errores". (Como comparando lo descrito con los alumnos de su grupo, agrega) estos niños, si usted observa un libro de primer año, qué hay en el libro, ipuros garabatos! y - según los niños - ahí dice papaya, manzana y no es correcto, por eso los maestros nos desesperamos. PD25-181102. Obs. Cl. + Entr. Ma. Macrina.

Desde la perspectiva de los nuevos materiales es esperable que los niños, al escribir por sí mismos, puedan producir escrituras que sean 'puros garabatos'; pero cómo y por qué actividades de este tipo pueden ser de utilidad para niños que todavía no saben escribir y, más aún, pueden ser de utilidad para que aprendan a escribir, son situaciones que resultan inicial- 
mente poco claras para los maestros. La escasa claridad sobre los sentidos y significados de dichas actividades para la enseñanza y el aprendizaje del lenguaje escrito explica, en parte, la desesperación y la incertidumbre que las maestras del estudio relatan haber experimentado durante sus primeras experiencias de trabajo con esta propuesta.

Fullan y Stiegelbahuer (1997), sostienen que, ante los procesos de cambio, los sentidos y significados para la enseñanza y el aprendizaje de los nuevos recursos van siendo construidos por los maestros durante el proceso mismo de su implementación. En un sentido semejante, las maestras del estudio refieren haber descubierto sobre la marcha cómo y para qué utilizar los recursos previstos por el PRONALEES. En este proceso, tanto las reuniones mensuales de trabajo convocadas por Nora, como las visitas que hacía al aula, según las describen las maestras, funcionaron como espacios de acceso a las propuestas de la PALEM y el PRONALEES. En la interacción propiciada por la participación conjunta en tareas específicas de planeación, evaluación y enseñanza fluyó información que alimentó la construcción de sentidos y saberes sobre los nuevos recursos propuestos. Perla, por ejemplo, señala cómo las reuniones les permitían resolver dudas y construir y compartir soluciones relativas al uso de los materiales.

En las reuniones aprovechábamos, exponíamos nuestras ideas y retomábamos las ideas de otros. Nora nos daba participación y nos dejaba proponer. Nos estimulaba que ella nos dijera: "miren la compañera le hizo así y así". Y ya tomábamos la idea de la compañera. Nos hacía sentir que nos estábamos dirigiendo bien, que íbamos por buen camino. [...] había bromas, pero eran comentarios de los niños, de lo que ellos hacían, nos reíamos. Luego nos acordábamos: "oye te acuerdas que en el examen de lectura decía la vaca come pasto y le preguntamos si decía pasto, y la niña dijo: sí allá en el campo" - risas-. Y esos comentarios pues también nos servían. PD14-021002 Obs. cl. + Entr. Ma. Perla.

A continuación utilizamos una descripción de Laura sobre la interacción que sostuvo con Nora en el desarrollo de una actividad de la PALEM durante una de sus visitas. Se muestra cómo en estas participaciones conjuntas Nora medió - aportando información - en la comprensión de Laura sobre la utilidad de uno de los recursos promovidos primero por la PALEM y después en el PRONALEES: la lectura de textos conocidos en los inicios de la alfabetización.

Laura inició como maestra suplente durante cuatro años. El último año, relata, se angustió cuando le asignaron el primer grado en una colonia marginal. No tenía experiencia con primero y no sabía cómo iba alfabetizar a los niños. Inicialmente, comenta, "también llevaba la idea de que les iba a poner planas, de la a, de la e”. Más temor sintió cuando le informaron que trabajarian con una nueva propuesta, la PALEM, y que tendría una asesora. Algunas de las maestras de su zona escolar no aceptaron involucrarse, ella en cambio comenta que aceptó porque Nora le inspiró confianza, le dijo "no te preocupes, vamos a aprender juntas”. Además, Laura consideraba que las planas eran tediosas para los niños; pero aunque rechazaba las planas, Laura, como muchos maestros, no tenía claro cómo es que los niños aprenderían a leer y escribir con las herramientas de la PALEM. En diversas ocasiones comentó sobre el desconcierto que le generaban las sugerencias de trabajo que le hacía Nora. No obstante, comenta, el diálogo con la asesora durante sus visitas, le permitió comprender las maneras en que determinadas actividades aportaban al aprendizaje de los niños:

En una ocasión recuerdo que la asesora nos llevó un verso, acerca de un conejito. Los niños lo cantaban y luego fueron señalando las palabras. Yo pensé: "para qué le va a servir eso al niño. O sea, yo dije: ay no, ipara qué hacer eso!" -admirada. Ya 
después le digo a Nora: "oye y ¿por qué vamos a hacer esa actividad?". Ella me dijo: "ah, es que con esa actividad el niño va a conocer la direccionalidad de la lectura, y también se va a familiarizar en que son letras y que dicen aaalgo". Hasta ese momento yo comprendí, dije: "ah, pues sí, realmente sí, al niño lo va a familiarizar más con la lectura". Pero primero, o sea cuando la maestra lo puso y me dijo vamos a hacer esto, yo dije: "y ¿para qué me va a servir a mí? ¿para qué va a ayudar a mis alumnos?” - mostrando asombro, sorpresa y duda -, o sea yo así lo pensé. Pero ya después, cuando la maestra me dio una explicación, y me dijo que servía para que el niño viera cuál era la direccionalidad de la lectura, y que aparte de eso lo iba a familiarizar con el sistema pues de la lectoescritura, que iban a distinguir que eran letras y que dicen algo; dije: "ah, no, pues tiene razón". Por ejemplo, en ese tiempo nosotros estábamos en una colonia, se podía decir que era un medio rural, donde muy pocas veces el niño tiene contacto con la lectoescritura antes de ingresar a la primaria. Entonces, en sí a los niños los motiva, los familiariza más con el sistema de la lectoescritura. Pero le digo, llegó un momento en que yo dije para qué les va a servir a mis alumnos que vayan a señalar las palabras, si ni saben leer, yo pensé pues ni saben leer - recuerda como divertida por lo que pensaba. 0 sea así lo pensé - como admirada”. PD15-091002 Entr. Ma. Laura.

Leer a los niños, o más aún que ellos intenten hacer por sí mismos la lectura de un texto conocido cuando todavía "ni saben leer", es una proposición de trabajo que, al igual que el escribir, resulta incomprensible para muchos maestros, por ello Laura, como denota en su relato, se mostraba sorprendida de que la asesora le propusiera la lectura de un verso y que los niños hicieran la lectura del mismo al tiempo que señalaban el escrito. Puesto su interés en que los niños aprendan a leer y escribir, para los maestros resulta fundamental tener claridad respecto a lo que aportan en este sentido los recursos que se les proponen. Laura, por ejemplo, asombrada por la actividad que Nora le sugería se preguntaba “¿por qué vamos a hacer esa actividad? ¿Para qué le va a servir eso al niño? ¿¡Para qué me va a servir a mí!? ¿Para qué va a ayudar a mis alumnos?”

La interacción descrita por Laura permite inferir que colegas más familiarizados con las nuevas propuestas de trabajo median en el acceso de los maestros a las mismas por la información particular y específica propiciada por la participación conjunta en tareas concretas.

De la interacción descrita es posible inferir, en primer lugar, que, con sus explicaciones enmarcadas en la realización de una actividad conjunta, Nora fue mediadora en el acceso de las maestras a los sentidos sobre la utilidad que podían tener para el aprendizaje los nuevos recursos. Laura, por ejemplo, apoyada en las explicaciones de Nora, parece encontrarle sentido a la lectura de textos propuesta en la PALEM y el PRONALEES como un recurso útil para el aprendizaje de los niños del lenguaje escrito. Así, tras explicarle Nora que leer primero a los niños y después pedirles que ellos intenten leer por sí mismos mientras van señalando el texto es una actividad que les permite conocer algunos aspectos que caracterizan al lenguaje escrito como "la direccionalidad de la lectura" y que las letras "dicen algo", Laura, según relata, parece encontrarle sentido a que los niños realicen esta actividad aún cuando "ni saben leer": “digo ‘ah, pues sí, realmente sí, al niño lo va a familiarizar más con la lectura”.

En segundo lugar, la descripción presenta indicios de que en la interacción con los colegas los maestros también reformulan sus propias ideas acerca de lo que es importante que los niños conozcan para lograr determinados aprendizajes. Laura, por ejemplo, haciendo uso de la información proporcionada por Nora, pareció modificar su idea de que el acceso de 
los niños a la lectura de textos tiene sentido sólo después de la adquisición del código alfabético. Apoyada en las explicaciones de la asesora encuentra que hay otros aspectos de la lectura - "direccionalidad", "que las letras dicen algo" -, que también importan para su aprendizaje y que sólo pueden "conocerse" mediante el involucramiento de los niños en la lectura misma de los textos.

En tercer lugar, en la descripción destaca el carácter situado y activo de la construcción de significados en la apropiación. Las explicaciones de Nora no son "lecciones acerca de la práctica", sino que "forman parte de y tienen lugar en ella" (Wenger, 2001). Se produjeron durante la puesta en juego de un recurso particular con un grupo de niños específico. En este marco Laura pareciera participar activamente en la producción de significados al relacionar los nuevos recursos con las explicaciones de Nora y las condiciones particulares de su grupo. Ella relacionó las explicaciones de Nora con las condiciones de vida y las posibilidades de contacto con el lenguaje escrito que tenían los niños con los que trabajaba cuando recibió la asesoría. Describe el lugar como un medio casi rural "donde muy pocas veces el niño tiene contacto con la lectoescritura". Reflexionando sobre estas condiciones de los niños con los que trabajaba, a partir de la información que Nora le proporcionó, Laura pareció encontrar significativo y pertinente realizar con sus niños esas actividades: dado su escaso "contacto con la lectura", resulta conveniente leer a los niños e involucrarlos en la realización de la lectura pues "los niños se familiarizan con la lectura”, al mismo tiempo que conocen sobre la misma aspectos tales como su "direccionalidad" y que lo escrito remite a significados, "las letras dicen algo".

El análisis precedente sugiere que en paralelo a la construcción de sentidos sobre los aportes de nuevas herramientas para el aprendizaje las maestras del estudio también reelaboraron sus ideas acerca de la enseñanza, el aprendizaje y el contenido que se enseña. Esta reelaboración, sin embargo, no antecedió al empleo en la práctica de las nuevas herramientas, sino que tuvo lugar en el proceso mismo de su implementación. Como mostraremos enseguida, un proceso similar tuvo lugar en relación con la producción de saberes prácticos relativos al empleo de los nuevos recursos en el aula.

\section{Mediación social y circulación y construcción de saberes prácticos}

El uso de determinados materiales para la enseñanza, supone una complejidad mayor que el mero seguimiento de un instructivo o manual. Contrario a una perspectiva técnica y racionalista, Schön (1992), sostiene que las prácticas profesionales están pobladas de situaciones singulares que los prácticos enfrentan produciendo conocimientos particulares y específicos en la acción. En este sentido, Lave (2001), entre otros, ha argumentado que la actividad, los cursos a seguir, se construyen relacionalmente entre las personas, las herramientas y el entorno, en la situación. En el marco de estas ideas, concebimos la apropiación de nuevas propuestas, la incorporación de sus recursos a la práctica, como un proceso donde los maestros construyen saberes prácticos relativos a la manera de emplearlos en las condiciones particulares y específicas que ellos enfrentan cotidianamente.

Nos apoyamos en la noción de "saberes docentes” de Mercado (1994; 2002); quien los define como un saber histórico y social sobre la enseñanza que los maestros construyen y reelaboran cotidianamente en la resolución diaria de su trabajo. Al denominarlo práctico, en este trabajo, se busca destacar el aspecto ligado a la acción de los saberes producidos por los maestros en la apropiación de nuevas propuestas pedagógicas.

El saber práctico es de naturaleza implícita y no proposicional, más que formulado se halla imbricado en la acción, en los sucesos particulares a los cuales sirve (Schön, 1992; Olson, 1992). Las dificultades que por ello entrañan los saberes prácticos para su comu- 
nicación y empleo en la formación de los profesionales, han sido documentadas por varios autores (Hiebert; Gallimore; Stigler, 2002; Olson, 1992; Schön, 1992; Tardiff, 2004). No obstante, en la realización cotidiana de las prácticas las personas emplean vías propias mediante las cuales participan de la construcción y circulación de esos saberes (Lave; Wenger, 2003). En el caso de la enseñanza, Talavera (1992), documentó la existencia de "redes" informales sostenidas por los maestros mediante las cuales fluye y se alimenta la producción social de saberes docentes. En un sentido similar, las descripciones de las maestras de nuestro estudio sugieren que el acceso a los saberes prácticos vinculados al empleo de nuevas propuestas tiene lugar en el desarrollo de la práctica misma, y que los colegas y personas más informadas sobre las nuevas propuestas posibilitan unos para otros el acceso a este saber mediante vías como las sugerencias, los relatos de experiencias, y la coparticipación y la observación de clases. Analizamos a continuación la clase de saber práctico que se construye y circula mediante una de estas vías, los relatos de experiencia (RE).

Entre los maestros, el intercambio de experiencias de trabajo es altamente valorado. Hay estudios que señalan estos intercambios como el aspecto más importante y valorado por los maestros de los cursos de actualización, así como su preponderancia en la construcción de sentidos sobre los contenidos y actividades de tales cursos (García, 2002). Las maestras del estudio también refieren el intercambio de experiencias como un recurso fundamental en la apropiación de nuevas propuestas pedagógicas.

En este estudio llamamos RE a las descripciones espontáneas de las maestras para comunicar y compartir con los colegas alguna experiencia de trabajo concreta. Los RE se articulan en torno de situaciones y preocupaciones docentes comunes, como en este caso lo es la experimentación de recursos novedosos para la alfabetización de los niños. Las maneras particulares en que cada maestro instrumenta los recursos y lo que de ello les resulta, son parte constitutiva de los RE, en este sentido constituyen un medio para la circulación de saberes prácticos relacionados con la puesta en juego de los recursos provenientes de nuevas propuestas.

Las referencias de las maestras a los intercambios de experiencia sugieren que ellas valoran los RE de sus colegas porque les ofrecen pistas, caminos que ellos pudieran seguir en su propio trabajo ante preocupaciones comunes. Nora recuerda que en las reuniones de planeación y evaluación, la preocupación compartida de los maestros por el avance de los niños en el aprendizaje de la lectoescritura propiciaba el intercambio espontáneo de experiencias y de materiales. Los maestros, narra, se mostraban interesados por conocer la forma de trabajo de los colegas que reportaban avances en el aprendizaje de sus alumnos:

Confrontábamos las gráficas y eso permitía que ellas se intercambiaran materiales. Por ejemplo, se decían: “a ver cómo están tus niños, itu grupo cómo está!, ¡huy amiga ya estás bien avanzada!". Y otras se quedaban realmente angustiadas, preocupadas por ver cómo estaban en la evaluación. Luego se preguntaban: “¿oye tú cómo le hiciste?”. Y ahí es donde se daba el intercambio [...] por ejemplo, los que ya habían avanzado, decían: "mira yo le saqué materiales a esto, yo hice esto, iyo inventé esto!" [...] Y luego les preguntaban: "oye ¿y te lo pudieron hacer los niños?”. PD53-280304 Entr. As. Nora.

Según narra Nora, las maestras describían para sus colegas las preocupaciones que se planteaban y las formas en que procedían. Como el caso de una maestra que les explicaba cómo es que ella trabajó con una pequeña rima para que los niños pudieran avanzar en el descubrimiento de las relaciones entre lo oral y lo escrito:

Recuerdo una maestra que decía: "dije cómo le voy a hacer, cómo le voy a hacer -tono de angustia. Entonces opté por seleccionar nada más las palabras grandes para quitarles las 
vocales e íbamos leyendo, yo lo leía en el pizarrón y los niños veían la letra que yo borraba, veían dónde se quedaban los espacios. Como yo llevaba copias, lo quité del pizarrón y les dije, 'ahora saben qué, ustedes vean qué cosas le hacen falta. Escriban lo que le falta'. Y cuando terminaban les decía que leyeran". Les decía a las demás maestras: "claro que los niños no saben leer pues, no conocen las letras, pero van haciendo el recorte sonoro". PD53-280304 Entr. As. Nora.

Como sugiere el comentario de Nora, los RE parecen contribuir a la apropiación de nuevas propuestas en tanto que proporcionan una perspectiva acerca de la forma en que determinados recursos pueden ser empleados ante situaciones o necesidades específicas y comunes. Apoyados en sus RE los maestros se comunican alternativas posibles de acción y recursos que pueden ser de utilidad ante determinadas situaciones; en ellos describen situaciones concretas en las que han probado su pertinencia y viabilidad de emplearlos y de utilizarlos en formas determinadas. Esto es, más que por las sugerencias de recursos que conllevan, los RE parecen ser valorados por los saberes prácticos articulados y puestos en acción en la experiencia descrita. Como se verá enseguida, estos saberes no son de naturaleza abstracta a manera de principios derivados de alguna formulación teórica, sino que se encuentran incorporados en las acciones particulares que se describen; se hallan integrados y se ponen de manifiesto en el sutil tejido relacional que se va configurando a partir del uso de un recurso específico, la intermediación de los maestros y la participación de los estudiantes en la actividad descrita.

El comentario de Laura sobre uno de los intercambios sostenidos con una de sus colegas de segundo año permite apreciar algunos de los saberes que circulan mediante los RE. Su relato se centra en las maneras en que se puede aprovechar la heterogeneidad del grupo de modo que todos los niños puedan beneficiarse con la realización de las actividades. El intercambio tiene lugar a propósito de la planeación conjunta por grados que se había acordado realizar en la escuela. Una de sus colegas, comenta Laura, se sentía preocupada porque consideraba que no podía avanzar en el programa debido a que sus alumnos aún no aprendían a leer y escribir.

Inicialmente Laura sugería a su colega trabajar con el nombre propio, actividad a la que en otras ocasiones ella se refirió como un recurso que emplea mucho durante el primer año porque ayuda a los niños en el aprendizaje del lenguaje escrito. Laura relató:

La maestra me comentó que los niños no saben leer ni escribir y, por ejemplo, yo le decía: "que formen su nombre, que formen nombres de otros niños". Dice ella: "pero por ejemplo esa actividad cómo la hago implicando que es algo imposible de que lo hagan los niños-, eso no lo van a saber hacer, si no saben leer, ni escribir". PD15091002 Entr. Ma. Laura.

Según comenta Laura, su colega consideraba que la formación de nombres no era una actividad accesible para sus niños. En varias conversaciones Laura manifestó que ella misma, en sus experiencias iniciales con la PALEM, se sorprendía que la asesora le recomendara actividades similares cuando sus alumnos aún no sabían leer y escribir. Quizá por ello, apoyándose en sugerencias específicas y RE, Laura pareciera no sólo intentar responder a la pregunta de su colega de cómo hacer la actividad, sino también ponerle de manifiesto cómo la mediación del maestro en esas actividades es fundamental para que los niños las realicen, sin que ello implique que dejen de trabajar y de aprender:

Yo le sugerí que trabajara en forma general, que hiciera el trabajo en el pizarrón, pero con las participaciones de los niños, porque, le dije: "yo siento que no todos 
van a estar en ese nivel, habrá algunos que ya sepan escribir, entonces ya con esos puedes hacer la actividad. También se pueden formar por equipos". Le dije a la maestra: "por ejemplo yo tengo tres niños que están en el nivel más bajo del grupo. Cuando siento que aquella actividad es muy difícil, les digo háganlo por parejas, y trato de sentar a ese niño con otro que ya puede hacer esa actividad. Entonces les digo a los niños, tú le vas a ayudar, no hacérselo, le vas a explicar. Y también cuando siento que una actividad es muy difícil, la hacemos todos en forma general, lo hago en el pizarrón, pero los niños van dando las ideas; y ya del pizarrón lo hacen ellos en su libro". 0 sea, no lo hago yo, no, es trabajo de ellos.

Le ponía el ejemplo de la invención de rimas. En el libro daban un ejemplo y los niños tenían que formar dos. Pensé que posiblemente sólo podrian tres o cuatro niños. Entonces las hicimos entre todos. Primero los formé por equipos y cada equipo lo hizo a su idea. Yo les preguntaba: "a ver tú cómo crees que empieza, y qué sigue”. Después presentaron la rima en el grupo y entre todos le fueron ayudando a mejorarla. Como se iban a escribir dos rimas en el libro, les dije que iban a escribir las que más les gustara. Yo no podía decir que escribieran alguna rima, para que el niño no viera que había preferencias. [...] Y de esa manera hicimos la actividad, pero el niño no va a decir la hizo la maestra o la hizo él, sino el grupo, la hicieron entre todos ellos. Entonces es lo que le sugería, que trabajara de esa manera para que no se quedara tanto. PD15091002 Entr. Ma. Laura.

Como se puede apreciar, Laura construye relatos de su experiencia docente relacionados con una situación específica que su colega le expone como problema para avanzar en los contenidos del programa de español: el hecho de que sus alumnos de segundo grado todavía no sepan leer y escribir. Recurriendo a su experiencia Laura le propone alternativas de trabajo "para que no se quedaran tanto", alternativas que ella emplea cuando, en situaciones similares a la que le refiere su colega, considera que sus alumnos no pueden realizar por sí mismos los contenidos y actividades previstas en el programa.

En su RE Laura articula diversos saberes relativos al empleo de los nuevos recursos en situaciones similares a la que enfrenta su colega. Así, por una parte, Laura destaca cómo organizar la realización colectiva de la actividad aprovechando la heterogeneidad de niveles de aprendizaje de los niños, y, por otra, cómo el maestro puede mediar en la actividad apoyando a los niños en su realización.

Respecto a los saberes relativos a la organización del trabajo, Laura describe formas de organización que ha implementado, las cuales promueven la colaboración de los niños en la realización de tareas que son complejas para algunos de ellos o para la generalidad del grupo. En este sentido, los saberes prácticos expresados se refieren a cómo suscitar la colaboración entre los niños, de modo que unos y otros se apoyen en la realización de la tarea y aprendan de la misma. Se trata de saberes en los que se ponen en relación la complejidad de las actividades sugeridas como herramientas para la enseñanza en los materiales curriculares y las posibilidades de los niños para realizarlas por ellos mismos. En este sentido, Laura comunica en su RE cómo ante las relaciones variables entre las tareas a realizar y las posibilidades de los niños para desarrollarlas, las formas de colaboración pueden organizarse de formas diversas. Por ejemplo, expresa Laura, cuando considera que la actividad es accesible para la mayoría de los niños, a los que podrían tener dificultad para realizarla los sienta con niños que sí pueden hacer la actividad, como en el caso de los tres alumnos "que están en el nivel más bajo del grupo". En otras ocasiones, cuando considera que la actividad podría ser compleja para la mayoría de los niños, la realiza de manera grupal; primero todos participan aportando ideas, las 
cuales retoma Laura para la resolución de la actividad en el pizarrón. En otros momentos, la colaboración se puede organizar y estimular como ayuda, después de que los niños realizan sus propios esfuerzos para resolver las tareas, tal como relata Laura que lo hizo en la actividad de redacción de rimas: "primero [...] cada equipo lo hizo a su idea. [...] después en el grupo entre todos le fueron ayudando a mejorarla".

Por otra parte, Laura expone en su RE saberes relativos a las formas y los sentidos de la mediación de la maestra en la realización de la actividad. En éstos se ponen de manifiesto algunas de las formas de intervención empleadas por Laura con el objeto de promover la colaboración entre los niños de forma que ésta contribuya a la resolución de las actividades al mismo tiempo que aliente el aprendizaje. Al igual que con la organización del trabajo, los saberes relativos a la intermediación en la actividad, son de carácter relacional, por lo que las formas de intervención contenidas en el relato de Laura varían de una situación a otra de las que ella describe. En ocasiones, la intervención de la maestra puede reducirse a sugerir cómo desarrollar la actividad conjuntamente, como cuando Laura sienta por parejas a un niño que puede hacer la actividad con otro que tiene dificultades para hacerlo: "les digo a los niños, tú le vas a ayudar, no hacérselo, le vas a explicar”. En otras ocasiones cuando la complejidad de la actividad se considera alta para la generalidad del grupo, la intervención puede ser más permanente y centrada en estimular las intervenciones de los niños necesarias para la realización de la tarea. Por ejemplo, cuando Laura relata la elaboración de las rimas primero en los equipos, pone de manifiesto cómo en ese caso ella interrogaba a los niños. A través de preguntas, por un lado, estimulaba la participación de los niños y, por otro, buscaba orientar las participaciones en un sentido que apoyaran la realización de la actividad: "a ver tú cómo crees que empieza, y qué sigue”.

Los RE de los maestros son más que simples anécdotas. Son un recurso del que ellos se valen para comunicar saberes sobre la enseñanza que construyen con la puesta en juego de determinados recursos. Aunque apoyadas en los recursos sugeridos en las propuestas curriculares, las experiencias relatadas son más que la descripción de su aplicación. La puesta en juego de un determinado recurso, como lo sugiere el RE de Laura sobre la elaboración de rimas, supone invención y descubrimiento; por ejemplo, en el relato de Laura, la forma más apropiada de organización del trabajo y los modos de intervención más pertinentes de la maestra en el desarrollo de la actividad para que los niños se involucren, aprendan de su realización y se aproveche en el proceso la heterogeneidad de niveles de aprendizaje de los niños. En este sentido, los relatos de experiencias aún cuando se basan en los recursos planteados en las nuevas propuestas curriculares, son más que eso, en ellos se expresan los recursos-más, más los saberes producto de la invención y los descubrimientos generados durante su implementación ${ }^{4}$.

\section{La apropiación y la capacitación docente: algunas conclusiones}

Indagando en el trabajo y las experiencias de maestras que valoran y emplean para la alfabetización inicial de sus alumnos la propuesta pedagógica del PRONALEES, el análisis precedente destaca aspectos importantes acerca de la apropiación de las reformas curriculares, particularmente de los soportes sociales que median en este proceso.

En primer lugar el análisis revela la apropiación de nuevas propuestas como un proceso

4. "Formar subgrupos en los que haya niños con distintos niveles de conocimiento, para que los más adelantados apoyen a sus compañeros", por ejemplo, es una de las proposiciones centrales en el PRONALEES y en varias de las situaciones didácticas se hacen recomendaciones al respecto. Lo distintivo en el RE de Laura es su recuperación como recurso para abordar los contenidos del programa que resultan inaccesibles para los niños, así como las formas de organización de la actividad y de intermediación de la maestra que hacen del "apoyo a los compañeros" un recurso que efectivamente contribuya al aprendizaje. 
de producción de nuevos sentidos y saberes situado en la enseñanza ${ }^{5}$. La incorporación del PRONALEES implicó para las maestras la reelaboración de sentidos sobre la enseñanza. No obstante, aunque indispensables, estas reelaboraciones no fueron previas sino producidas socialmente en la acción, como sucedió con la maestra Laura cuando la asesora le propuso y argumentó la conveniencia de que los niños leyeran un texto conocido. Las dudas sobre la utilidad, sobre los sentidos de los distintos recursos del PRONALEES y las respuestas a las mismas, tuvieron lugar en la realización de la enseñanza, en el involucramiento conjunto de las maestras en tareas tales como la planeación, la evaluación, la solución de problemas específicos o la conducción de clases particulares.

En segundo lugar, el análisis destaca cómo, simultáneo a la construcción de sentidos, los maestros producen saberes prácticos. Los maestros no reproducen fielmente las nuevas propuestas, aunque crean en ellas, las transforman en su uso al considerar las particularidades de sus alumnos. De esta forma producen los saberes prácticos necesarios para hacer de los nuevos recursos herramientas efectivas para el aprendizaje, como lo muestra Laura en su RE sobre la organización de la clase y las modalidades de intervención docente ante la complejidad de las actividades sugeridas y las posibilidades de los niños para resolverlas en forma independiente.

Finalmente, de manera central, en el análisis destaca la necesaria mediación social en el acceso a las nuevas propuestas. En la interacción con la asesora y los colegas, propiciada por la participación conjunta en tareas propias de la enseñanza, circuló información particular y específica que, estrechamente articulada a las dudas y las dificultades enfrentadas en la acción, alimentó la producción de sentidos y saberes relativos a los nuevos recursos.

En resumen, la apropiación del PRONALEES, desde la perspectiva de las maestras de este estudio, se muestra como un proceso que tiene lugar en la acción, en el que paulatinamente los maestros, en interacción con personas más informadas y con sus colegas, producen sentidos y saberes prácticos necesarios sobre los recursos propuestos. Visto de esta manera, parece necesario replantear algunas de las ideas y acciones actuales que guían los esfuerzos por acercar a los maestros al conocimiento y empleo en sus prácticas de las reformas curriculares.

En primer lugar, parece necesario situar la capacitación de los maestros en la práctica de la enseñanza. El reconocimiento de que los maestros poseen conocimientos, creencias y saberes que son determinantes en la configuración de sus prácticas, se ha traducido en diversos programas de capacitación en un esfuerzo por conducir a los maestros a su reformulación en un sentido congruente con las nuevas propuestas. Bajo el supuesto de que en posesión de nuevas concepciones los maestros interpretarán e implementarán de manera coherente los cambios curriculares, pocas veces se contemplan en estos espacios oportunidades para la puesta en juego de las nuevas informaciones en la realización de tareas concretas donde se espera que los maestros efectúen cambios. En algunos estudios, sin embargo, se documenta la dificultad que para los maestros reviste el transitar de proposiciones abstractas y generales a su instrumentación en el aula para resolver situaciones específicas (Espinosa, 2002; Lerner, 2001). Si, como sugiere este artículo, los maestros reelaboran sus ideas tanto como sus saberes prácticos situados en la implementación misma de las reformas, entonces el involucramiento de los maestros en procesos de planeación, evaluación, búsqueda y solución conjunta a problemas particulares de enseñanza, así como la posibilidad de obser-

5. En algunos trabajos como el de Putnam y Borko (2000), lo situado se restringe a la localización espacial, a los espacios físicos como el aula. En nuestra perspectiva lo "situado" alude más bien a la inserción del aprendizaje en el marco de una práctica social como lo es la enseñanza. En este sentido los aprendizajes sobre el PRONALEES logrados por las maestras del estudio estuvieron situados por el involucramiento de ellas en la realización conjunta de distintas tareas pertenecientes a la enseñanza, aún cuando algunas de éstas como la planeación y el análisis de evaluaciones tuvieron lugar fuera del salón de clases. 
varse en clases concretas, deben ocupar un espacio central en las acciones de capacitación.

La formulación de materiales curriculares ricos en descripciones didácticas detalladas, como los del PRONALEES, son un medio apropiado, pero insuficiente para la incorporación de las nuevas propuestas a las aulas. Los maestros necesariamente elaboran sentidos sobre sus aportes para el aprendizaje y las formas particulares en que pueden ser incorporadas a partir de la realidad concreta de sus aulas. Y este proceso, como se desprende del análisis presentado, es de naturaleza social, se alimenta de la circulación de información, materiales, experiencias probadas que se propicia con la participación conjunta de maestros involucrados en la experimentación de las nuevas propuestas. Desde esta perspectiva, pareciera más conveniente alentar y fortalecer en los espacios de capacitación la conformación de estructuras de participación y trabajo colectivo entre los maestros centradas en la planeación y evaluación de la enseñanza. Ello implica aceptar que los maestros producen nuevos saberes durante la implementación y no sólo aplican las reformas, asimismo implica dar cabida a las vías de mediación social que los maestros de por sí emplean para apoyarse mutuamente.

Finalmente, el análisis sugiere que la asesoría como medio para la implementación de reformas curriculares puede contribuir a su apropiación por parte de los maestros mediante la creación de comunidades de práctica (Wenger, 2001). Los comentarios de las maestras del estudio muestran una historia de aprendizaje compartido. Interactuando a través del tiempo en la realización conjunta de tareas como la planeación, la evaluación y la realización de clases, ellas fueron reelaborando visiones compartidas acerca de la enseñanza y el aprendizaje iniciales del lenguaje escrito. Simultáneamente, las maestras fueron creando un repertorio de estrategias y saberes compartidos sobre las maneras de implementar en el trabajo del aula la nueva propuesta. Ambas caracteristicas definen lo que es una comunidad de práctica y muestran su importancia para el desarrollo profesional de los maestros, tanto como para la incorporación de nuevas perspectivas a las prácticas cotidianas de enseñanza.

\section{Referencias bibliográficas}

AVALOS, A. Estudio de las transformaciones que sufren las concepciones de los maestros sobre contenidos geométricos en un curso de actualización. 1996. Tesis (Maestría)- DIE-CINVESTAV. México, 1996.

AVILA, A. Los profesores y sus representaciones sobre la reforma a las matemáticas. Perfiles Educativos, v. 23, n. 93, 2001. p. 59-86.

BAJTIN, M. Teoría y estética de la novela. Madrid: Taurus, 1989.

CARVAJAL, A. El uso de un libro de texto visto desde la etnografía. Revista Mexicana de Investigación Educativa, v. 6, n. 12, 2001. p. 223-247.

COLE, M. Psicología cultural. Madrid: Morata, 1999.

COLE, M.; ENGESTRÖM, Y. Enfoque histórico-cultural de la cognición. En: SALOMÓN, G. (Comp.) Cogniciones distribuidas: consideraciones psicológicas y educativas. Buenos Aires: Amorrortu, 2001. p. 23-74.

CHARTIER, R. Sociedad y escritura en la edad moderna. México: Instituto Mora, 1995.

ESPINOSA, E. Un programa de formación docente en su realización cotidiana: una aproximación etnográfica. Tesis (DIE)- DIECINVESTAV, México, 2002. 
ESPINOSA, E. et al. La asesoría técnico pedagógica a los maestros en servicio: retos y logros en el PRONALEES. México, 2003. (Reporte de investigación inédito)

EZPELETA, J. Innovaciones educativas: reflexiones sobre los contextos en su implementación. Revista Mexicana de Investigación Educativa, v. 9, n. 21, 2004. p. 403-424.

FULLAN, M.; STIEGELBAUER, S. El cambio educativo: guía de planeación para maestros. México: Trillas, 1997.

GARCÍA, M. El papel de la experiencia docente en los cursos de capacitación para maestros multigrado. 2002. Tesis (Maestría)- DIE-CINVESTAV. México, 2002.

GEERTZ, C. La interpretación de las culturas. México: Gedisa, 1987.

HARGREAVES, A. Profesorado, cultura y postmodernidad (cambian los tiempos, cambia el profesorado). Madrid: Morata, 1996.

HIEBERT, J.; GALLIMORE, R.; STIGLER, J. W. A knowledge base for the teaching profession: what would it look like and how can we get one? Educational Researcher, v. 31, n. 5, 2002. p. 3-15.

HUTCHINS, E. El aprendizaje de la navegación. En : CHAIKLIN, S.; LAVE, J. (Coords.) Estudiar las prácticas: perspectivas sobre actividad y contexto. Buenos Aires: Amorrortu, 2001. p. 49-77.

LAVE, J. La práctica del aprendizaje. En: CHAIKLIN, S.; LAVE, J. (Coords.). Estudiar las prácticas: perspectivas sobre actividad y contexto. Buenos Aires: Amorrortu, 2001. p. 15-46.

LAVE, J.; WENGER, E. Aprendizaje situado: participación periférica legítima. México: UNAM-FESI, 2003.

LERNER, D. Leer y escribir en la escuela: lo real, lo posible y lo necesario. México: FCE, 2001.

MERCADO, R. Saberes and social voices in teaching. En: ALVAREZ, A.; del Rí0, P. (Eds.). Education as cultural construction. Madrid: Fundación Infancia y Aprendizaje y Universidad Complutense, 1994. p. 61-68.

Los saberes docentes como construcción social: la enseñanza centrada en los niños. México: FCE, 2002.

OLSON, J. Understanding teaching: beyond expertise (Developing teachers and teaching). Londres: Open University Press, 1992.

PEA, R. Prácticas de inteligencia distribuida y diseños para la educación. En: SALOMÓN, G. (Comp.). Cogniciones distribuidas: consideraciones psicológicas y educativas. Buenos Aires: Amorrortu, 2001. p. 75-125.

ROCKWELL, E. Reflexiones sobre el proceso etnográfico. México: IPN-CINVESTAV-DIE, 1987.

ROCKWELL, E.; MERCADO, R. La práctica docente y la formación de maestros. En: docente: descripciones y debates. México: IPN-CINVESTAV-DIE, 1986. p. 63-75.

La escuela, lugar del trabajo

SCHÖN, D. La formación de profesionales reflexivos: hacia un nuevo diseño de la enseñanza y el aprendizaje en las profesiones. Barcelona: Paidós, 1992.

SEP. Libro para el maestro. Español primer grado. México: SEP, 1998.

TALAVERA, M. L. Construcción y circulación social de recursos docentes en primer grado. Estudio etnográfico. 1992. Tesis (DIE)- CINVESTAV-IPN-DIE, México, 1992.

TARDIFF, M. Los saberes docentes y su desarrollo profesional. Madrid: Narcea, 2004.

TATTO, M. T. Para una mejor formación de maestros en el México rural: retos y tensiones de la reforma constructivista. Revista Mexicana de Investigación Educativa, v. 4, n. 7, 1999. p. 101-136.

TYACK, D.; CUBAN, L. En busca de la utopía: un siglo de reformas en las escuelas públicas. México: SEP, 2000. 
VYGOTSKI, L. S. El desarrollo de los procesos psicológicos superiores. Barcelona: Grijalbo, 1988.

VIÑA0, A. La cultura de las reformas escolares. Perspectivas docentes, n. 26, 2001. p. 38-66.

WENGER, E. Comunidades de práctica: aprendizaje, significado e identidad. Barcelona: Paidós, 2001.

Recibido en 20.05.08

Aprobado en 17.03 .09

Epifanio Espinosa Tavera es Doctor en Ciencias en la Especialidad de Investigaciones Educativas (DIE-CINVESTAV), es profesor e investigador de la Universidad Pedagógica Nacional, Unidad 12B.

Ruth Mercado Maldonado es Doctora en Ciencias en la Especialidad de Investigaciones Educativas (DIE-CINVESTAV), es profesora e investigadora del Departamento de Investigaciones Educativas del Centro de Investigación y de Estudios Avanzados. 